\title{
COLLAPSE WARNING SYSTEM USING LSTM NEURAL NETWORKS FOR CONSTRUCTION DISASTER PREVENTION IN EXTREME WIND WEATHER
}

\author{
Chih-Chiang WEI (10* \\ Department of Marine Environmental Informatics \& Center of Excellence for Ocean Engineering, \\ National Taiwan Ocean University, 20224 Keelung, Taiwan
}

Received 17 July 2020; accepted 3 March 2021

\begin{abstract}
Strong wind during extreme weather conditions (e.g., strong winds during typhoons) is one of the natural factors that cause the collapse of frame-type scaffolds used in façade work. This study developed an alert system for use in determining whether the scaffold structure could withstand the stress of the wind force. Conceptually, the scaffolds collapsed by the warning system developed in the study contains three modules. The first module involves the establishment of wind velocity prediction models. This study employed various deep learning and machine learning techniques, namely deep neural networks, long short-term memory neural networks, support vector regressions, random forest, and $k$-nearest neighbors. Then, the second module contains the analysis of wind force on the scaffolds. The third module involves the development of the scaffold collapse evaluation approach. The study area was Taichung City, Taiwan. This study collected meteorological data from the ground stations from 2012 to 2019. Results revealed that the system successfully predicted the possible collapse time for scaffolds within 1 to $6 \mathrm{~h}$, and effectively issued a warning time. Overall, the warning system can provide practical warning information related to the destruction of scaffolds to construction teams in need of the information to reduce the damage risk.
\end{abstract}

Keywords: wind forecasting, machine learning, construction engineering, collapse warning, extreme weather.

\section{Introduction}

Taiwan experience typhoons frequently, because it is situated near the Asian continent and on the main paths of western North Pacific typhoons (Cheng et al., 2008). In Taiwan, scaffold collapse has serious consequences. For example, on September 27, 2016, Typhoon Megi made landfall in Taiwan and caused the scaffolds on the external walls of Le Méridien Taichung to collapse (Figure 1). The collapse of the scaffolds resulted in disruption of traffic in the surrounding area, the crushing of numerous vehicles, and the injury of various victims (Lin \& Yen, 2016). The purpose of this study is to develop a warning system for determining whether a scaffold would be damaged by extreme wind velocity (e.g., typhoons) and how to effectively reduce occupational accidents caused by the collapse of scaffolds.

In Taiwan, frame-type scaffolding is the most common scaffolding method used at construction sites. The existing Taiwan's laws related to the assembly requirements for frame-type scaffolds can refer to Ministry of Labor
[MOL] (2014) and Ministry of the Interior [MOI] (2014). As noted in Lin and Yen (2016), frame-type scaffolds are temporary structures for construction projects that are immediately dismantled after use. The risk of collapse is often underestimated. MOL (2014) stated that when façade frame type scaffolds are assembled at the outer area of the buildings, they often collapse under two conditions. First, they could collapse during the construction. Second, they could collapse when the construction is shut down due to the typhoon invasion.

Strong wind during extreme weather conditions is one of the natural factors that causes the collapse of frametype scaffolds. Therefore, an alert system is needed; such a system should be able to evaluate if scaffolding can withstand the effect of the wind force and to predict the possible future destruction time points on a real-time basis; this would provide construction teams with more advanced alert. The purpose of this study was to develop a typhoon-wind-induced scaffold-collapse early warn-

*Corresponding author. E-mail: ccwei@ntou.edu.tw 
a)

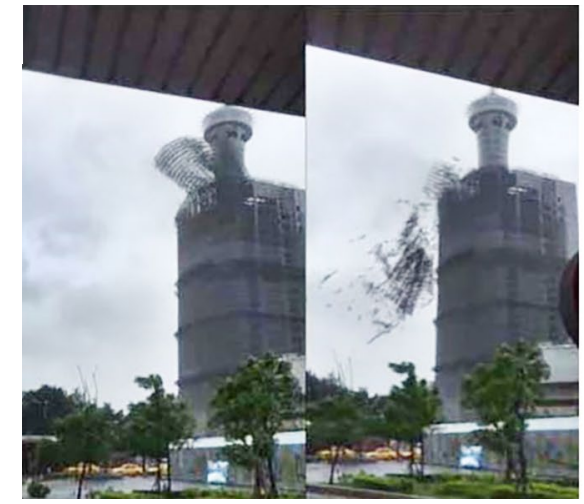

b)

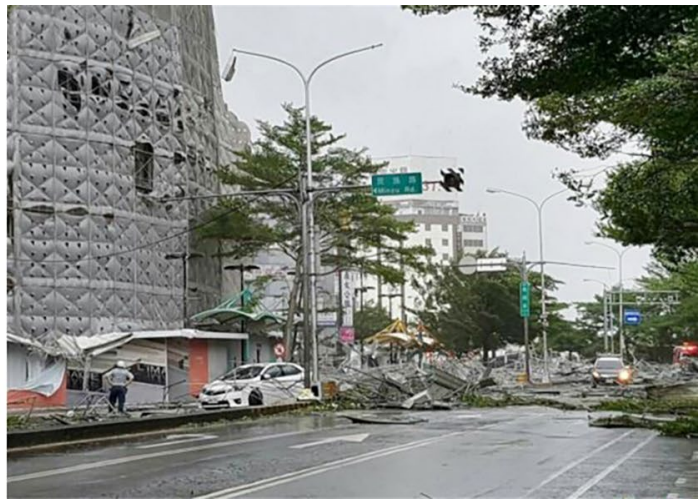

Figure 1. Scaffold collapse in Le Méridien Taichung

ing system (TISCEWS) for determining whether scaffold structures can withstand the stress of the wind force. For real-time operation, extreme wind force prediction is required for the TISCEWS system to simulate the possible destruction time point for frame-type scaffolds. Because accurate wind velocity prediction is necessary for operating real-time warning systems, this study established wind velocity prediction models by using various deep learning (DL) and machine learning (ML) techniques.

This study reviewed the relevant literature on wind velocity prediction models using DL-based and MLbased approaches, because they exhibit high calculation efficiency and accurate prediction ability (Dongmei et al., 2017; Mallick et al., 2020; Panapakidis et al., 2019; Sheela \& Deepa, 2013; Wei, 2014, 2015). In addition, several popular approaches are investigated such as autoregressive integrated moving average (Cadenas \& Rivera, 2010; Cadenas et al., 2016), support vector machine (Chou et al., 2020; Wei, 2017), random forest (Kim et al., 2019), radial basis function (Noorollahi et al., 2016), and neural networks (Chen et al., 2018; Huang et al., 2018a; Hu et al., 2016; Wei, 2019). A recurrent neural network (RNN) is an extension of a conventional feedforward neural network that can handle a variable-length sequence input (Chung et al., 2014; Graves, 2013). RNNs are connectionist models with the ability to selectively pass information across sequence steps while processing sequential data at a time (Lipton et al., 2015). The RNN handles the variable-length sequence by having a recurrent hidden state whose activation at each time is dependent on that of the previous time (Chung et al., 2014; Graves, 2012).

RNNs have already exhibited fair performance in resolving time-series-related problems (Glüge et al., 2014; Zhang et al., 2019). RNNs, however, have difficulty capturing long-term memories; the gradient-vanishing problem generated in the calculation process of RNNs causes the long-term memories to be hidden by the shortterm memories. To solve the problem of vanishing gradient in traditional RNNs, Hochreiter and Schmidhuber (1997) proposed the long short-term memory (LSTM), which introduces a structure called a memory cell to develop persistent long-term dependencies. The overall effect of the gate units is that the LSTM memory cells can store and access information over long periods of time and thus avoid the vanishing gradient problem (Chung et al., 2014; Wollmer et al., 2010). Therefore, much attention has been given to the LSTM in the processing of time-series problems (Byeon et al., 2015; Lu et al., 2019; Weninger et al., 2014). In the development of wind velocity predictions, results of relevant studies such as Huang et al. (2018b), Huang and Kuo (2018), Liu et al. (2018), and Shi et al. (2018) have indicated that the LSTM could generate excellent prediction results for wind velocity prediction. To model the time series of wind speed data, this study used the LSTM neural networks for the prediction of typhoon wind speeds because of their appropriate long- and short-term time dependency management. Consequently, this study applied DL-based approaches, namely deep neural network (DNN) and LSTM, to construct the wind velocity prediction model. Moreover, conventional ML approaches such as support vector regression (SVR), random forest (RF), and $k$-nearest neighbors $(k \mathrm{NNs})$ were used as benchmark models. These approaches were compared to ensure the timeliness and accuracy of real-time velocity predictions.

\section{Study area and data}

The study area is Taichung City (Figure 2) located in the core zone between southern and northern Taiwan. It has an area of $2215 \mathrm{~km}^{2}$ and a population of over 2.8 million. Taichung City is currently the second largest city in Taiwan. Due to the rapid growth of its population, 48 buildings with heights above $120 \mathrm{~m}$ have already been built in the city.

This study collected data about the typhoon events for which typhoon alerts were issued by the Central Weather Bureau (CWB) and further selected typhoon events that affected the city. From 2012 to 2019, a total of 18 typhoon events (Table 1) affected Taichung City. Figure 3 illustrates the paths of these typhoons. Additionally, the wind velocity data from ground stations in Taichung City and its surrounding areas.

Figure 4 plots the maximum wind values of all typhoons recorded at the 21 ground stations at Taichung City. The wind velocity data were collected consisted of 


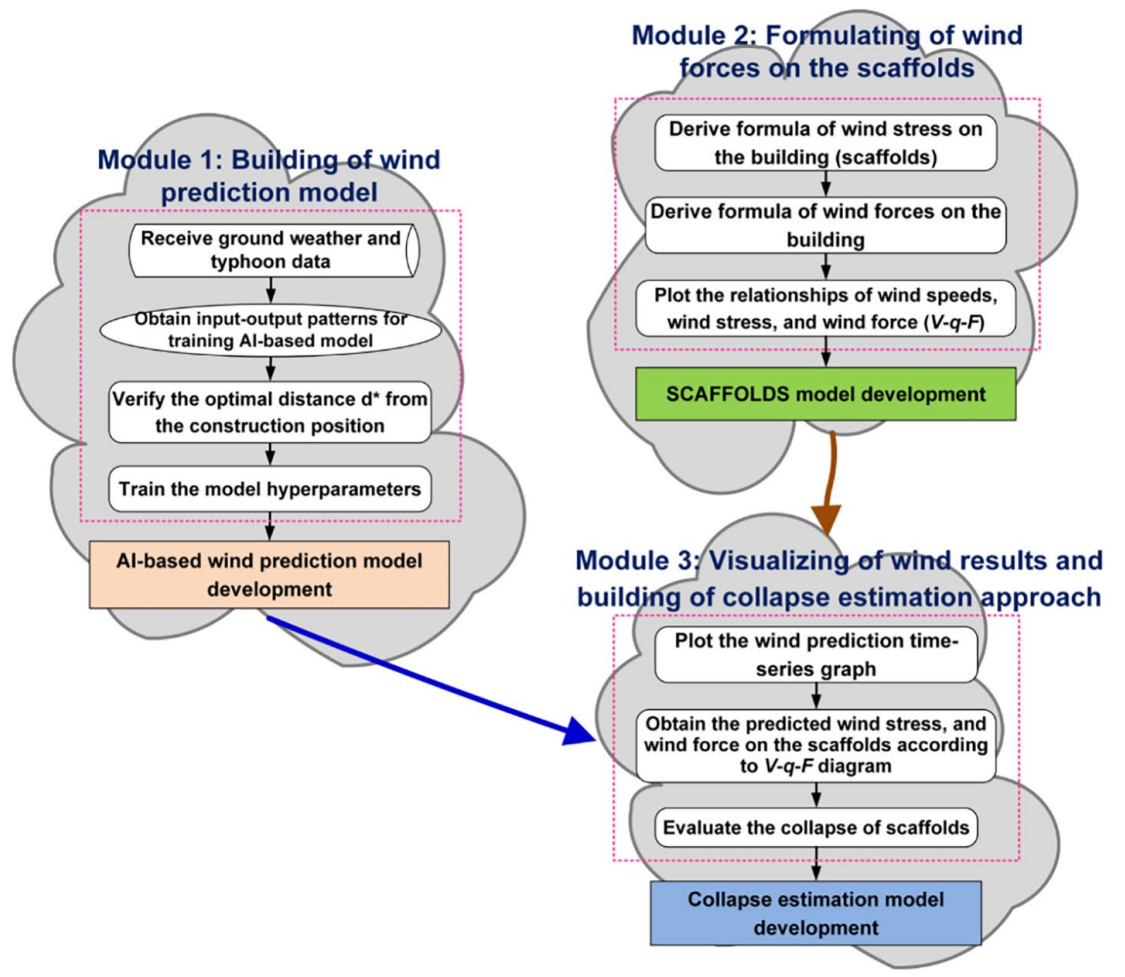

Figure 2. Flowchart of the methodology of SCTWWS

a)

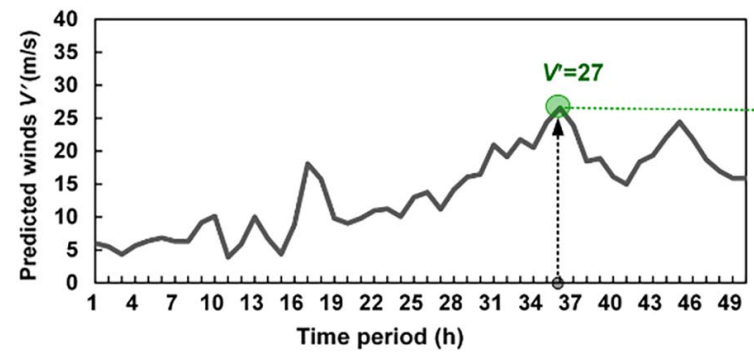

b)

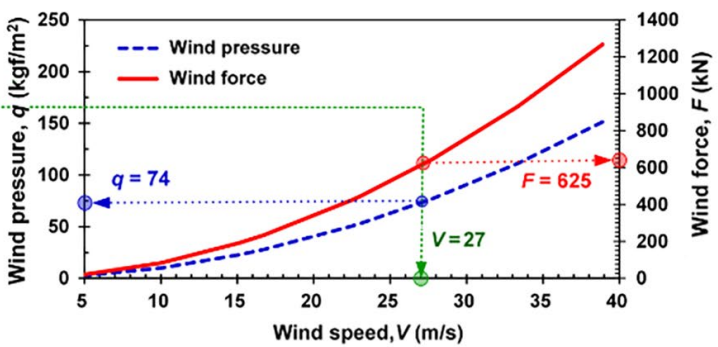

Figure 3. Visualized wind prediction results and scaffold collapse evaluation approach

Table 1. Typhoons affecting Taichung and surroundings from 2008 to 2019

\begin{tabular}{|l|c|l|c|}
\hline \multicolumn{1}{|c|}{ Typhoon } & Period & \multicolumn{1}{c|}{ Typhoon } & Period \\
\hline Fung-Wong & 27-28 Jul 2008 & Matmo & 19-23 Sep 2014 \\
\hline Morakot & 7-8 Aug 2009 & Fung-Wong & $6-10$ Aug 2015 \\
\hline Fanapi & 18-19 Sep 2010 & Soudelor & 27-30 Sep 2015 \\
\hline Nanmadol & 28-29 Aug 2011 & Dujuan & 6-10 Jul 2016 \\
\hline Talim & 19-22 Jun 2012 & Nepartak & 12-16 Sep 2016 \\
\hline Saola & 30 Jul-3 Aug 2012 & Meranti & 25 Sep 2016 \\
\hline Tembin & 21-29 Aug 2012 Jul 2017 \\
\hline Soulik & 11-14 Jul 2013 & Megi & 30 Jul-1 Aug 2017 \\
\hline Trami & 20-23 Aug 2013 & Haitang & $23-26$ Aug 2019 \\
\hline Kong-Rey & 27-30 Aug 2013 & Bailu & 29 Sep-2 Oct 2019 \\
\hline Fitow & $4-8$ Oct 2013 & Mitag & \\
\hline
\end{tabular}

the 10 -minute average wind velocity values measured at $10 \mathrm{~m}$ above ground. In the figure, the station with code 467770 (Wuqi station) recorded the highest wind velocity values (as high as $33.5 \mathrm{~m} / \mathrm{s}$ ) of all stations; the average wind velocity values of Wuqi station (as high as $5.4 \mathrm{~m} / \mathrm{s}$ ) were also the highest of all stations. The Wuqi station is close to Taichung Harbor in a developing area of Taichung City, and it contains numerous buildings under construction. Therefore, Wuqi station was selected as the experimental location. 


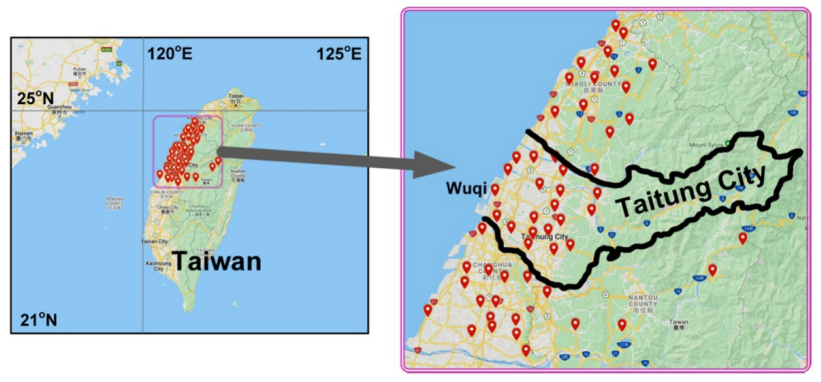

Figure 4. Geographical location of Taichung City

\section{Methodology}

The TISCEWS warning system developed in the study contains three modules for use in determining whether the scaffold structure could withstand the stress of the wind force. Figure 5 illustrates the flowchart of the system.

Each module is as follows.

Module 1: Typhoon wind prediction model. Various algorithms (including DL-based and ML-based approaches) were used to construct the typhoon wind velocity prediction model. The procedures for building the prediction model entailed the collection and analysis of typhoon data, preprocessing of data, development of the DL-based and ML-based models, output generation, and comparison of the prediction results (as described in Section 2.1).

Module 2: Scaffold wind force analysis model. This module (known as the SCAFFOLDS model) presented a method for calculating the wind force capable of being sustained by temporary buildings, i.e., scaffolds. The SCAFFOLDS model consisted of the derivation of a wind force formula (including the design wind force, wind pres-

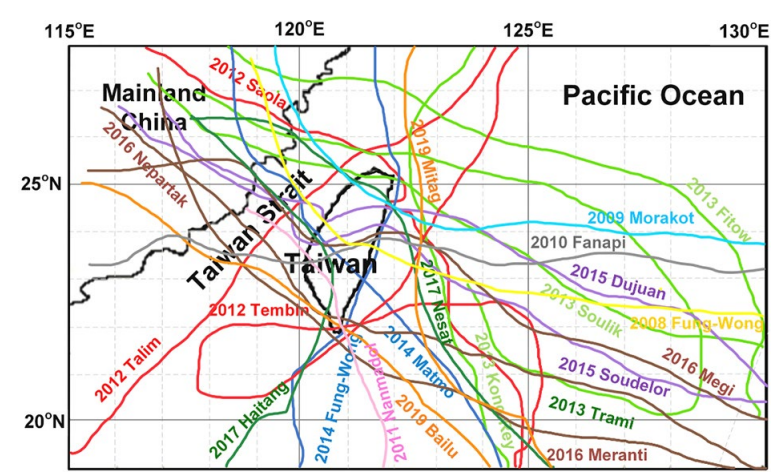

Figure 5. Historical typhoon tracks

sure, and basic design wind velocity of the buildings) and the estimation of scaffold wind-resistance strength during the construction periods (see Section 2.2). Finally, this module entailed plotting a relationship diagram of wind velocity $(V)$ - wind pressure $(q)$ - wind force $(F)$.

Module 3: Scaffold collapse evaluation model. The evaluation results were presented in a visualized manner to enable construction teams to rapidly and effectively comprehend whether the wind force may likely result in substantial damage. There are four steps:

- Step 1 is the visualization of wind prediction results. The wind velocity time-series diagram can be plotted (as illustrated in Figure 6a) on the basis of the predicted wind velocity data $\left(V^{\prime}\right)$ generated by Module 1 .

- Step 2 to determine the corresponding scaffold wind pressure $\left(q^{\prime}\right)$ and corresponding wind force $\left(F^{\prime}\right)$ under the predicted wind velocity. Take Figure 6 as an example: assume the aim is to evaluate whether destruction would occur at the 36 th hour at the current

a)



b)

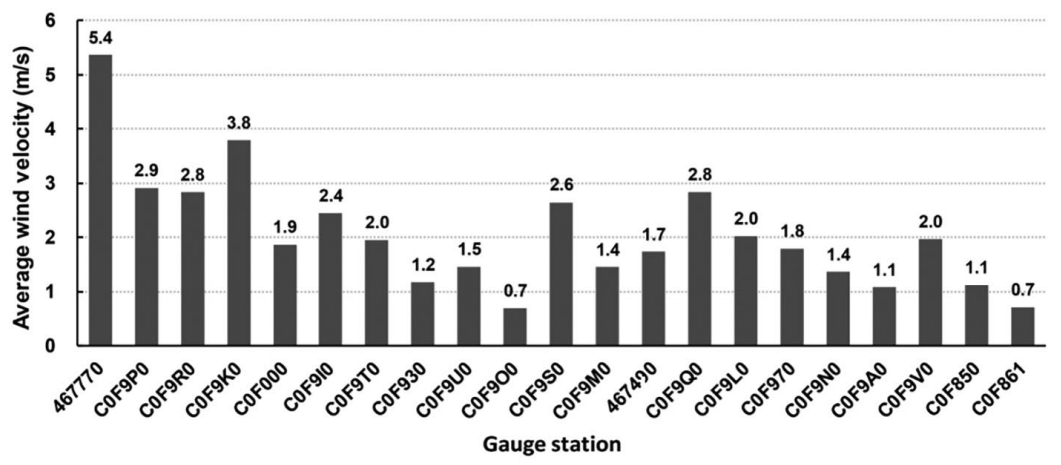

Figure 6. Wind speed records of typhoons 
time (i.e., the 35 th hour); the predicted wind velocity, $V^{\prime}=27 \mathrm{~m} / \mathrm{s}$, could be determined using Figure 6 a. Based on the predicted velocity determined using Figure $6 \mathrm{~b}$, it is inferred that the corresponding wind pressure is equal to $q^{\prime}=74 \mathrm{kgf} / \mathrm{m}^{2}$ and the corresponding wind force is equal to $F^{\prime}=625 \mathrm{kN}$.

- Step 3 is to estimate the scaffold's safe bearing stress $\left(\lambda_{\max }\right)$ and safe bearing capacity $\left(F_{\max }\right)$. In Taiwan, it is possible to extrapolate the theoretical safe bearing capacity that a scaffold should possess based on the design wind force calculation stipulated by MOI (2015).

- Step 4 is to evaluate whether the scaffolds are in the danger of collapsing. The scaffolds are safe if the predicted wind force $F^{\prime}$ is smaller than the scaffold's safe bearing capacity, $F_{\text {max }}$. If it is not, the scaffolds are not safe and are at risk of collapsing under the effect of wind force. Therefore, the system would issue a destruction warning.

In the following sections, the processes of constructing the DL-based and ML-based models and SCAFFOLDS model for the TISCEWS system are described.

\subsection{Development of wind prediction model}

\subsubsection{Theorem of algorithms}

The applied DL-based and ML-based approaches were briefly reviewed in this section. A DNN is a typical representative of feedforward artificial neural networks, and uses the backpropagation algorithm for training ( $\mathrm{Hu}$ et al., 2016). It consists of three parts: an input layer, multiple hidden layers, and an output layer (Huang et al., 2019). A DNN can extract features that are representative of data characteristics through linear or nonlinear transformation in multiple processing layers ( $\mathrm{Du} \& \mathrm{Xu}, 2017)$. The update of weight can be resolved using the stochastic gradient descent method represented by the following equation:

$$
\Delta w_{i j}(t+1)=\Delta w_{i j}(t)+\eta \frac{\partial c}{\partial w_{i j}},
$$

where $\eta$ is the learning rate, and $c$ is the cost function.

For the LSTM model, an LSTM layer is composed of multiple recurrently connected subnets, which are referred to herein as memory blocks (Wollmer et al., 2013). An LSTM network is the same as that of a standard RNN, except that the summation units in the hidden layer are replaced by memory blocks (Graves \& Schmidhuber, 2005). Figure 7 illustrates a single memory cell in an LSTM layer. Every memory block consists of self-connected memory cells $c_{t}$ and three multiplicative gate units (input gate $i_{t}$, output gate $o_{t}$, forget gate $f_{t}$ ). The input gate controls the flow of input activations to the memory cell. The output gate controls the output flow of cell activations to the rest of the network. The forget gate scales the internal state of the cell before adding it as the input to the cell through the self-recurrent connection of the cell, thereby adaptively forgetting the cell's memory (Sak et al., 2014). Eqns

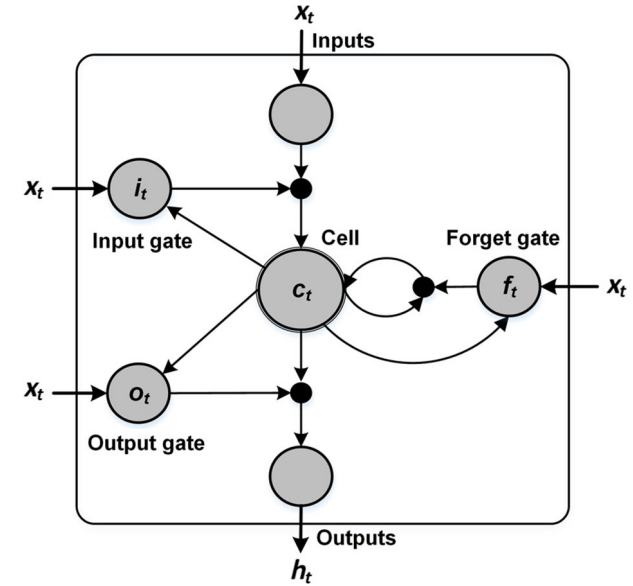

Figure 7. Architecture of a memory cell in an LSTM

(2) to (6) are used to compute the output of these units (Graves, 2012).

$$
\begin{aligned}
& f_{t}=\left(W_{f} x_{t}+U_{f} h_{t-1}+b_{f}\right) ; \\
& i_{t}=\left(W_{i} x_{t}+U_{i} h_{t-1}+b_{i}\right) ; \\
& o_{t}=\left(W_{o} x_{t}+U_{o} h_{t-1}+b_{o}\right) ; \\
& c_{t}=f_{t} c_{t-1}+i_{t}\left(W_{c} x_{t}+U_{c} h_{t-1}+b_{c}\right) ; \\
& h_{t}=o_{t}\left(c_{t}\right),
\end{aligned}
$$

where $x_{t}$ is the input vector; $\sigma$ is the activation function; $W_{f}, W_{i}, W_{o}, W_{c}, U_{f}, U_{i}, U_{o}$, and $U_{c}$ are the weight vector terms; $b_{f}, b_{i}, b_{o}$, and $b_{c}$ are the corresponding bias terms; and $h_{t}$ and $h_{t-1}$ are the current and previous hidden vectors, respectively.

SVR, which was developed by Vapnik (1995), is an algorithm based on statistical learning theory and is gaining popularity due to its many attractive features and promising generalization performance (Üstün et al., 2005). Support vector machines (SVMs) are developed for risk minimization, which reveals that the generalization error is bounded by the sum of training errors. This induction principle is based on the bounding of the generalization error by the sum of the training error and a confidence interval term depending on the Vapnik-Chervonenkis (VC) dimension. Thus, SVM achieves an optimum network structure by striking an appropriate balance between the empirical error and the VC-confidence interval (Chen \& Wang, 2007). SVMs have been extended to solve nonlinear regression estimation problems through techniques such as SVR, which exhibit excellent performance (Cristianini \& Shawe-Taylor, 2000; Wei, 2012).

$\mathrm{RF}$ is a bagging-based algorithm proposed by Breiman (2001). In the RF algorithm, classification trees are used. Each tree makes a class prediction based on various predictor variables. The trees are created using bootstrapping, a technique which holds back parts of the available objects and uses random subsets to grow various decision trees for each class (Brandt et al., 2014). Thus, the large number of trees reduces the generalization error (Baudron et al., 2013; 
Pal, 2005). Because the out-of-bag observations are not used in the fitting of the trees, the out-of-bag estimates can be used to perform cross-validation accuracy estimation (Cutler et al., 2007).

$k \mathrm{NN}$ is an unsupervised algorithm. Fix and Hodges (1951) proposed a classification method that is easy to implement and provided high accuracy. $k \mathrm{NN}$ is a nonparametric method for pattern classification based on determining the "nearest" training set (Masetic \& Subasi, 2016). Selecting the optimal number of neighbors $k$ first depends on the inspection of the specific data set. Therefore, the $k$ value is estimated using the available training sample observations (Beli \& Guo, 2017). Generally, $k$ is crucial to the $k \mathrm{NN}$ algorithm, and the accuracy of $k \mathrm{NN}$ classification is subject to the volume of the classified training data.

\subsubsection{Modeling process}

Figure 8 plots the maximum wind velocity values recorded of Wuqi station during typhoons. As shown, the highest values were recorded during Megi $(33.5 \mathrm{~m} / \mathrm{s})$, followed by Soudelor $(30.8 \mathrm{~m} / \mathrm{s})$ and Dujuan $(26.6 \mathrm{~m} / \mathrm{s})$. This study selected the top three wind velocity values as the testing set. Wind velocity data of the remaining 15 typhoons were used as the training and validation sets. Model training and validation were performed through 10 -fold crossvalidation.

The DL-based and ML-based models were used to build the rainfall prediction model. Before constructing these prediction models, this study identified the optimal weather data from adjacent stations that could effectively represent wind velocity prediction at Wuqi station. Figure 9a presents the root mean squared error (RMSE) results for different distance settings using $10 \mathrm{~km}$-interval. The RMSE indicator was defined as:

$$
\mathrm{RMSE}=\sqrt{\frac{1}{n} \sum_{i=1}^{n}\left(V_{i}^{\mathrm{pre}}-V_{i}^{\mathrm{obs}}\right)^{2}},
$$

where $n$ is the number of data entries, $V_{i}^{\text {pre }}$ is the $i$ th predicted value, and $V_{i}^{\text {obs }}$ is the $i$ th observed value.

A total of $3,12,24,33,44,49,52$, and 55 weather stations can be found within the distance range from 10 to $80 \mathrm{~km}$. As illustrated, the smallest RMSE values for the prediction models could be obtained when the distance was $50 \mathrm{~km}$. Therefore, this study employed meteorological data from adjacent 44 stations for the following various model constructions.

In the construction of the DL-based and ML-based models, the hyperparameters were calibrated through trial and error; that is, a single parameter was fixed, and another parameter was adjusted to verify the parameter combination with a low error. For the DL-based DNN and LSTM models, the adaptive moment estimation optimization algorithm (Adam optimizer) was used to optimize the momentum and learning rate when training the both models. The Adam optimizer proposed by Kingma and $\mathrm{Ba}$ (2015) can be used instead of the classical stochastic gradient descent procedure, because it can dynamically adjust the learning rate and update the weight of neural networks, ultimately improving calculation efficiency (Yao et al., 2017). Furthermore, this study adopted ReLU (Rectified linear unit) activation function, developed by Nair and Hinton (2010), in the middle layers (i.e., hidden layer and LSTM layer in both models), because it converges quickly and can conquers the problem of vanishing gradient. ReLU is defined as the positive part of its argument: $f(x)=\max (0, x)$, where $x$ is the input to a neuron. To avoid the presented model from being overfitted, a method called dropout can be used (Huang et al., 2019). A dropout rate of 0.2 is applied to the output of LSTM layer in the study. During the training of both model structures for lead time $=1 \mathrm{~h}$ (future $1 \mathrm{~h}$ ), the parameters of the number of hidden layers $\left(N_{\mathrm{hl}}\right)$ and the number of neurons in a hidden layer $\left(N_{\text {neu }}\right)$ were calibrated. First, the $N_{\mathrm{hl}}$ was calibrated using the default $N_{\text {neu }}=30$. Figure $9 \mathrm{~b}$ illustrates the RMSE results for the DNN and LSTM models for various $N_{\mathrm{hl}}$ values. Both models yielded the smallest RMSE values when the $N_{\mathrm{hl}}$ was equal to 1 . Then, the $N_{\text {neu }}$ was calibrated. Figure $9 \mathrm{c}$ illustrates the RMSE results for the DNN and LSTM models showing that the smallest $N_{\text {neu }}$ values are at 50 and 40 , respectively.

For ML-based models, the SVR, RF, and $k \mathrm{NN}$ model parameters for 1 -h lead prediction were calibrated. For SVR, this study used the radial basis function (RBF) as the kernel function because it is a popular kernel function used in various kernelized learning algorithms. Here, parameters cost $(C)$, epsilon $(\varepsilon)$, and gamma $(\gamma)$ were calibrated. Here, $C$ parameter denotes the trades off correct



Figure 8. Maximum wind velocity in each typhoon 
classification of training examples against the maximization margin of the decision function, $\varepsilon$ denotes the maximum absolute error to a specified margin, and $\gamma$ denotes the parameter of the RBF kernel. Figures 10a-10c display the process of the trial-and-error approach. First, the $C$ value was calibrated using the default $\varepsilon=0.001$ and $\gamma=0.01$.

After obtaining the optimal $C$ value $(=100)$, the subsequent parameter $\varepsilon$ was validated (fixed the optimal $C$ value and $\gamma=0.01$ ). When the optimal $\varepsilon$ was validated to be 0.03 , the $\gamma$ was validated. Finally, the optimal values were determined to be $C=100, \varepsilon=0.03$, and $\gamma=0.02$. When the RF model was constructed, the major parameters, size of each bag $\left(b_{\text {size }}\right)$ and maximal depth $\left(d_{\max }\right)$ were calibrated. First, the $b_{\text {size }}$ was calibrated using the default $d_{\max }=30$. After obtaining the optimal $b_{\text {size }}$, the parameter $\varepsilon$ was validated. Figures $10 \mathrm{~d}, 10 \mathrm{e}$ reveal that the optimal $b_{\text {size }}$ is 80 and $d_{\max }$ is 20 . In addition, when the $k \mathrm{NN}$ model was constructed, the number of neighbors $\left(N_{\mathrm{nb}}\right)$ were calibrated. Figure $10 \mathrm{f}$ reveals that the optimal $N_{\mathrm{nb}}$ was 40 . Using the same processing, this study performed parameter calibrations for a lead time of 2-6 $\mathrm{h}$ (a forecast horizon of 2-6 h). Table 2 lists the results of parameter calibrations for DL-based and ML-based models in lead time $=1-6 \mathrm{~h}$. Moreover, Figure 11 displays the RMSE values of the DL-based and ML-based models using parameter calibrations for lead times of $1-6 \mathrm{~h}$. a)

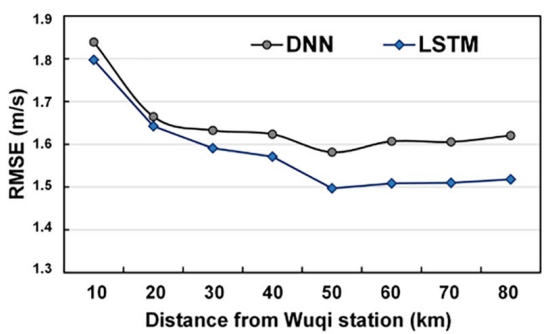

b)

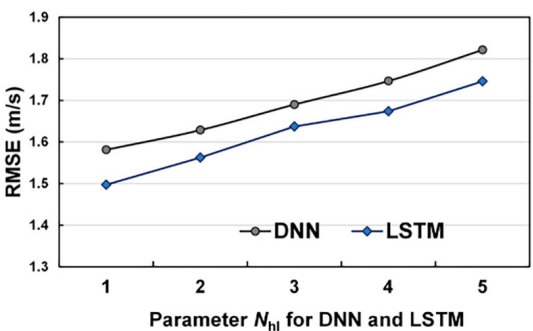

c)



Figure 9. Calibration of the suitable distance and parameter calibrations

a)

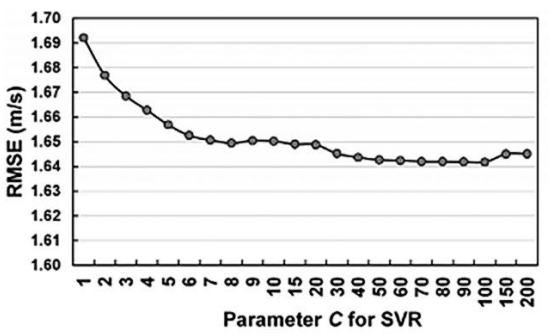

d)

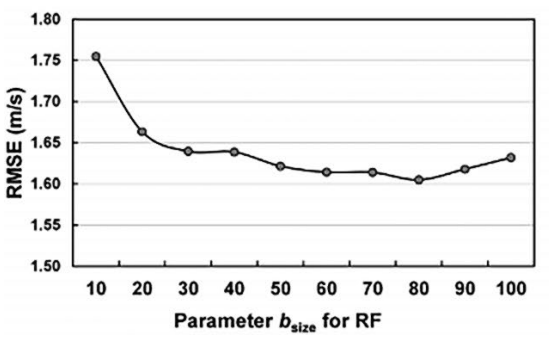

b)

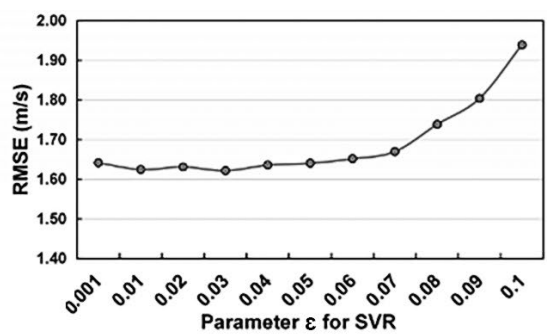

e)

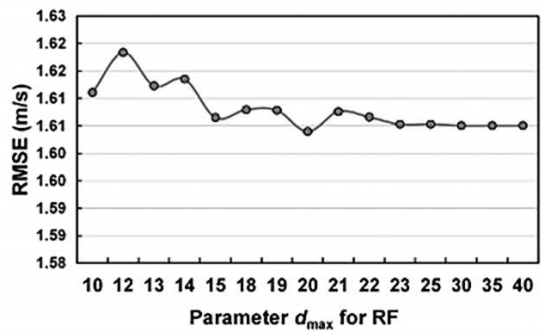

Figure 10. ML-based SVR, RF, $k N N$ c)

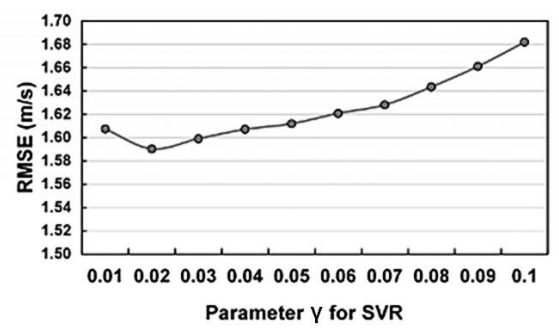

f)

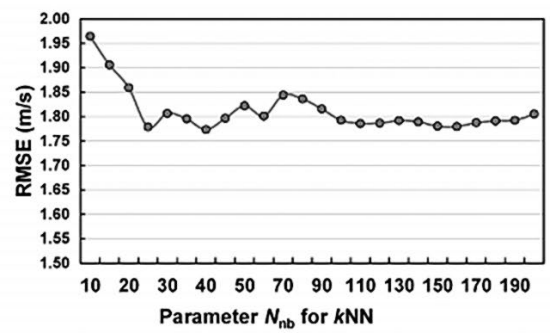

Table 2. Parameter calibrations for DL-based and ML-based models in lead time $=1-6 \mathrm{~h}$

\begin{tabular}{|c|c|c|c|c|c|}
\hline \multirow{3}{*}{ Lead time (h) } & \multicolumn{5}{|c|}{ Models and their parameters } \\
\hline & $\mathrm{DNN}$ & LSTM & SVR & $\mathrm{RF}$ & $k \mathrm{NN}$ \\
\hline & $\left(N_{\mathrm{hl}}, N_{\text {neu }}\right)$ & $\left(N_{\mathrm{hl}}, N_{\text {neu }}\right)$ & $(C, \varepsilon, \gamma)$ & $\left(b_{\text {size }}, d_{\max }\right)$ & $N_{\mathrm{nb}}$ \\
\hline 1 & $(1,50)$ & $(1,40)$ & $(100,0.03,0.02)$ & $(80,20)$ & 40 \\
\hline 2 & $(1,53)$ & $(1,41)$ & $(90,0.01,0.01)$ & $(70,25)$ & 45 \\
\hline 3 & $(1,56)$ & $(1,41)$ & $(80,0.03,0.03)$ & $(60,23)$ & 50 \\
\hline 4 & $(1,59)$ & $(1,44)$ & $(80,0.02,0.02)$ & $(60,27)$ & 50 \\
\hline 5 & $(1,70)$ & $(1,46)$ & $(90,0.03,0.04)$ & $(50,28)$ & 55 \\
\hline 6 & $(1,68)$ & $(1,43)$ & $(90,0.04,0.03)$ & $(50,30)$ & 60 \\
\hline
\end{tabular}




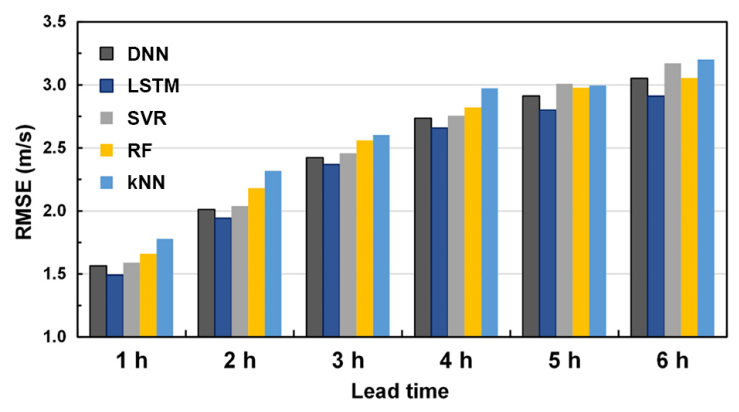

Figure 11. Performance levels of lead times 1-6 h

\subsection{Development of SCAFFOLDS model}

In this section, SCAFFOLDS is explained using the scaffold construction condition of Le Méridien Taichung as a case study.

\subsubsection{Design wind force of the building, $F$}

The Le Méridien Hotel Taichung has a total height of 145 $\mathrm{m}$. According to MOI (2015), the closed buildings refer to buildings that are enclosed by external walls, and open buildings refer to buildings that possess beam columns but not walls. Scaffolds of concern in the study belong to the open building category. According to the MOI (2015), the formula for calculating wind force for open buildings $(F)$ is:

$$
F=q\left(z_{A c}\right) \cdot G \cdot C_{f} \cdot A_{c},
$$

where $G$ refers to the gustiness response factor of the building and its function is to convert the average wind velocity into the gust wind velocity; $C_{f}$ is the wind force coefficient of wind effects; $A_{C}$ is the characteristic area of an open building subjected to the effect of wind; $z_{A c}$ is the centroid elevation of characteristic area $A c$; and $q\left(z_{A c}\right)$ refers to the wind velocity pressure of the height $z_{A c}$.

The $z_{A c}$ of the collapsed scaffolds of said building is $139 \mathrm{~m}$, and the characteristic area of the scaffolds $(A c)$ is approximately $600 \mathrm{~m}^{2}$. The value of parameters $G$ and $C_{f}$ in Eqn (8) were obtained from MOI (2015), and the values of $G$ and $C_{f}$ were 1.711 and 1.2, respectively. After substituting these parameters into Eqn (8), the design wind force $F$ can be obtained:

$$
F(z=139 \mathrm{~m})=1232 \cdot q\left(z_{A c}\right) \text {. }
$$

In Eqn (9), the unit of wind pressure $q(z)$ is $\mathrm{kgf} / \mathrm{m}^{2}$. Therefore, the unit of the design wind force $(F)$ would be kgf. Because the commonly used unit for force is $\mathrm{kN}$ $(1 \mathrm{kN}=1000 \mathrm{~N} ; 1 \mathrm{kgf}=9.8 \mathrm{~N})$, our formula for converting unit $F$ into unit $\mathrm{kN}$ is as follows:

$$
F(z=139 \mathrm{~m})=12.07 \cdot q\left(z_{A c}\right) .
$$

\subsubsection{Design wind pressure, $q(z)$}

The calculation of $q(z)$ in Eqn (10) is as follows (Lin \& Yen, 2017; MOI, 2015):

$$
q(z)=0.06 \cdot K(z) \cdot K_{z t} \cdot\left[I \cdot V_{10}(C)\right]^{2},
$$

where $K(z)$ refers to the ground condition coefficient of height $z$ under the wind pressure; $K_{z t}$ is the ground condition coefficient (represents the partial acceleration effect of wind velocity that occurs on the top half of a hill or mountain ridge as well as the peak near the cliff (generally, $K_{z t}$ can be set to 1 ); I refers to the occupancy coefficient (scaffolds are temporary facilities, and therefore, $I$ could be set to 0.9$)$; and $V_{10}(C)$ is the basic design wind velocity.

In Eqn (11), $K(z)$ is the function of the height from ground, as follows:

$$
K(z)=2.774 \cdot\left(\frac{z}{z_{g}}\right)^{2 \alpha} .
$$

In Eqn (12), $\alpha$ refers to the value of various ground conditions; and $z_{g}$ refers to the gradient elevation. Value of $\alpha$ and $z_{g}$ can be obtained from MOI (2015); their values are 0.15 and $300 \mathrm{~m}$, respectively. Therefore, $K(z=139 \mathrm{~m})$ is equal to 2.202. In Eqn (11), $V_{10}(C)$ is defined as the "the 10 minute average wind velocity (return period of 50 years), measured $10 \mathrm{~m}$ above ground (reference height) when the ground condition is C" according to MOI (2015); ground condition $C$ refers to open and wide land, coast, or shore area; ground condition $A$ refers to the city center of a metropolis, and ground condition $B$ refers to a suburban area of a metropolis or a small town). After substituting these parameters into Eqn (11), the wind pressure formula can be converted into the following:

$$
q(z=139 \mathrm{~m})=0.107 \cdot\left[V_{10}(C)\right]^{2} .
$$

Wuqi station is located near the coastal area, and thus, the ground condition of the station to be of the type $C$ ground condition was determined. According to MOI (2015), the corresponding $V_{10}(C)$ of Taichung City is $32.5 \mathrm{~m} / \mathrm{s}$. Therefore, when $V_{10}(C)=32.5 \mathrm{~m} / \mathrm{s}$, the corresponding design wind pressure is equal to $q(z=139 \mathrm{~m})=$ $113 \mathrm{kgf} / \mathrm{m}^{2}$, and the design wind force is equal to $F(z=$ $139 \mathrm{~m})=1364 \mathrm{kN}$.

\subsubsection{The design wind resistance strength of scaffolds}

Frame-type scaffolds belong to the temporary structures. Therefore, the scaffolds often cannot achieve the wind resistance standards stipulated for permanent buildings. In Taiwan, scaffolds can be assembled based on the following laws stipulated by MOI $(2014,2015)$ : "during the construction period, sufficient temporary support needs to be provided to resist the effect of wind force on the structural materials or parts"; "given that the temporary structures erected during construction period is used for a shorter period of time, basic design wind velocity $V_{10}(C)$ with a shorter return period could be used. However, the return period of $V_{10}(C)$ cannot be shorter than 10 years".

In the derivation of the formula for basic design wind velocity $V_{10}(C)$ shown previously, $V_{10}(C)$, with a return period of 50 years, was used. In accordance with the relevant laws, this study adopted a basic design wind velocity return period of 10 years for the scaffolds. According to MOI (2015), the wind velocity of a return period of 10 
years is 0.782 times the wind velocity of a return period of 50 years. Therefore, $V_{10}(C)_{\text {return }=10}=25.42 \mathrm{~m} / \mathrm{s}$. Therefore, the design wind pressure with a return period of 10 years could be set to $q(z=139 \mathrm{~m})_{\text {return }=10}=69.11 \mathrm{kgf} / \mathrm{m}^{2}$, whereas the wind resistance strength of the scaffolds (i.e., the design wind force of the scaffolds) is $F(z=$ $139 \mathrm{~m})_{\text {return }=10}=834 \mathrm{kN}$.

\section{Simulation and collapse evaluation}

The simulation enabled the establishment of a complete set of evaluation procedures suitable in the study site.

\subsection{Procedures for real-time operation of system}

Figure 12 illustrates the procedure for real-time operation of TISCEWS system. The simulation interval was set to 1 $\mathrm{h}$, and the forecasting horizon was 1 to $6 \mathrm{~h}$. The procedural steps are as follows:

Step1: Receive typhoon path information from the CWB.

Step2: Does the CWB issue a land warning for a typhoon? If yes, make time $=t$ and execute step 3; if not, return to step 1 .

Step3: Activate the TISCEWS system.

Step4: Collect real-time climate information, including meteorological data from the ground stations and typhoon events.
Step5: Collect information about the buildings under construction (e.g., the condition of the scaffold at the construction project site).

Step6: Execute the DL-based model (Module 1) and generate the wind velocity prediction values, $\left\{V^{\prime}{ }_{t+\Delta t}\right\}_{\Delta t=1,6}$.

Step7: Execute the SCAFFOLDS model (Module 2) and generate the $V-q-F$ relationship curve diagram.

Step8: Execute the scaffold collapse evaluation approach (Module 3); this step involves plotting of wind velocity graph based on the wind velocity values predicted in step 6 . Additionally, the $V-q-F$ relationship diagram from step 7 is used to determine the corresponding wind pressure $\left\{q^{\prime}{ }_{t+\Delta t}\right\}_{\Delta t=1,6}$ and wind force $\left\{F^{\prime}{ }_{t+\Delta t}\right\}_{\Delta t=1,6}$.

Step9: Estimate the safe bearing stress $\left(\lambda_{\max }\right)$ and the safe bearing capacity $\left(F_{\max }\right)$ of the scaffold structure.

Step10: Evaluate whether the scaffolding would be destroyed by the typhoon and whether a warning is needed within the next $6 \mathrm{~h}$. Use the destruction evaluation methods of Module 3 to evaluate whether the scaffolding would be destroyed and at what time. If yes, execute step 11. If not, execute step 12 instead.

Step11: Output information regarding the time of possible scaffold collapse $\left(t_{\text {collapse }}\right)$ and the warning

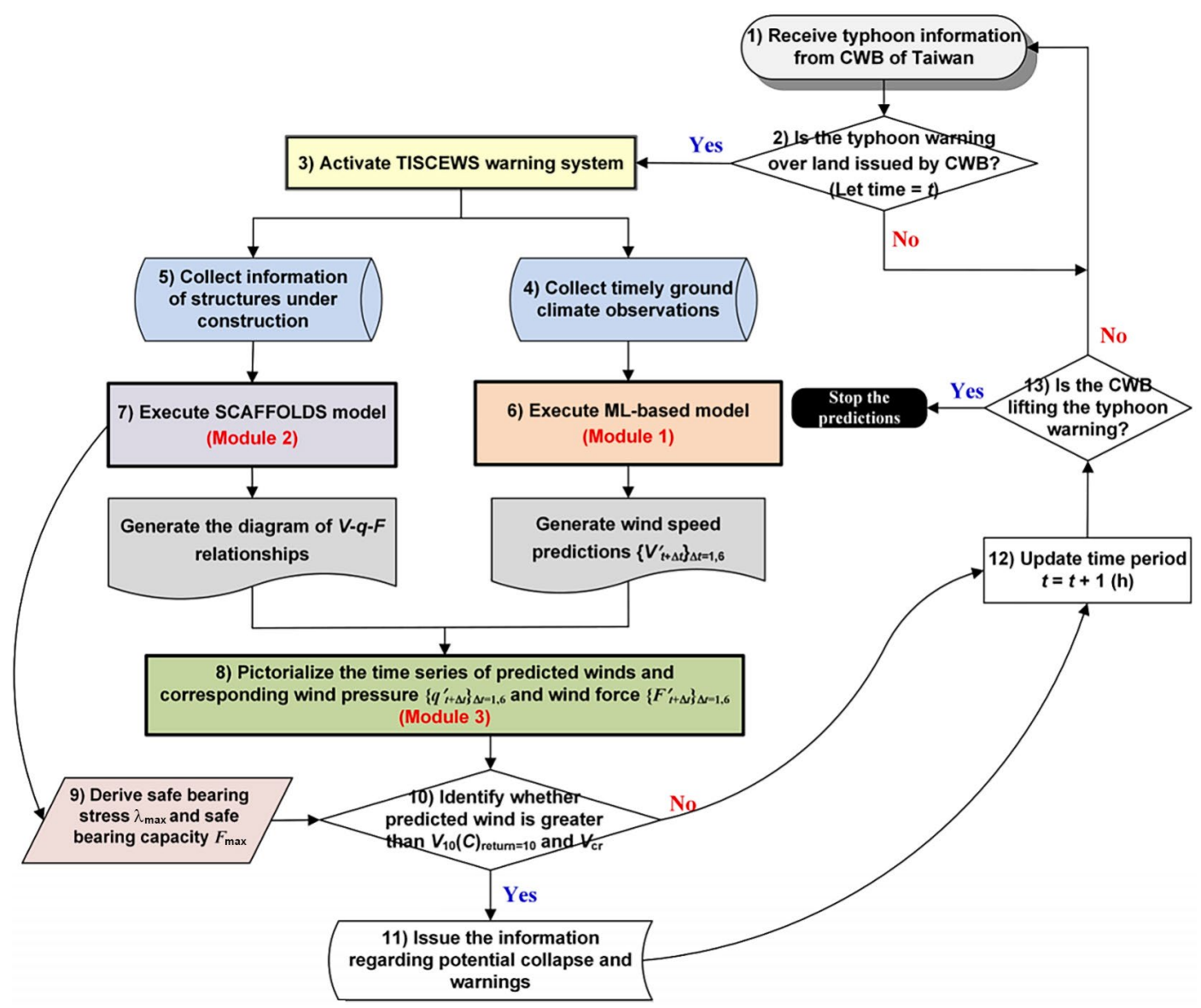

Figure 12. Concept flowchart of real-time operation 
time $\left(t_{\mathrm{cr}}\right)$ under the wind velocity alert value (warning time, $t_{\mathrm{cr}}$ ). The issued information includes the possible destruction time point for the scaffold structures $\left(t_{\text {collapse }}\right)$, the corresponding wind velocity $\left(V_{t_{\text {collapse }}}\right)$ and wind force $\left(F_{t_{\text {collapse }}}\right)$, the warning time $\left(t_{\mathrm{cr}}\right)$, and the corresponding wind velocity $V_{t_{\mathrm{cr}}}$ and wind force $F_{t_{\mathrm{cr}}}$.

Step12: Update the calculation time, $t=t+1$ (h).

Step13: Does the CWB lift the typhoon warning? If yes, end the analysis procedure. If not, return to step 1 and perform the prediction analysis for the next time period.

To test the usability of the TISCEWS, simulations were performed at the experimental site; these are described in the subsequent section.

\subsection{Wind velocity simulation results}

As mentioned earlier, three typhoon events (Soudelor in 2015, Dujuan in 2015, and Megi in 2016) were used as a test set. The prediction results for the upcoming 1 to $6 \mathrm{~h}$ for all test typhoon cases are presented in Figure 13. In each subfigure, the data for three typhoons are presented; on the time series of the $x$-axis, the first time point for Typhoon Soudelor is 01:00 on 2015/08/06 (simulated for a total of $114 \mathrm{~h}$ ), the first time point for Typhoon Dujuan is 01:00 on 2015/09/27 (90 h), and the first time point for Typhoon Megi is 01:00 on 2016/09/25 (114 h).

As illustrated in Figure 13, LSTM model generated data that were closer to the observed data than the DNN, $\mathrm{SVR}, \mathrm{RF}$ and $k \mathrm{NN}$ generated data. Additionally, as the forecasting horizon increased, peak wind velocity prediction ability decreased gradually. Figure 14 plots the results for RMSE indicator; LSTM model exhibited a smaller prediction error than that of the DNN, SVR, RF and $k \mathrm{NN}$ models. Because LSTM model exhibited significantly better performance than other models in generating predictions, the wind velocity prediction results generated using LSTM model were used as the input variables for Modules 2 and 3 in the subsequent scaffold destruction analysis.

\section{3. $V$-q-F diagram}

The $V-q-F$ relationship diagram was generated on the basis of the construction context conditions of the scaffolds of the Le Méridien Hotel Taichung case. For the sake of plotting the $V-q-F$ diagram (i.e., step 7 of the procedures shown in Figure 12), Eqns (13) and (10) can be used to obtain the $V$ - $q$ curve and $V-F$ curve:

$$
\begin{aligned}
& q^{\prime}(z=139 \mathrm{~m})=0.107 \cdot V^{\prime} ; \\
& F^{\prime}(z=139 \mathrm{~m})=12.07 \cdot q^{\prime}(z=139 \mathrm{~m}),
\end{aligned}
$$

where $V^{\prime}=$ predicted wind velocity (unit: $\mathrm{m} / \mathrm{s}$ ), $q^{\prime}$ is the corresponding wind pressure $\left(\mathrm{kgf} / \mathrm{m}^{2}\right)$ under the predicted wind velocity, and $F^{\prime}$ is the corresponding wind force $(\mathrm{kN})$ under the predicted wind velocity.

The $V-q-V$ relationship curve diagram (Figure 15) can be obtained using Eqns (14) and (15). Regarding the safe bearing stress of scaffolds $\left(\lambda_{\max }\right)$ and the safe bearing ca- pacity of scaffolds $\left(F_{\max }\right)$ in step 9 of Figure 12, a design wind pressure value $q(z=139 \mathrm{~m})_{\text {return }=10}$ was calculated according to the laws stipulated by MOI (2015) as the safe bearing stress $\left(\lambda_{\max }\right)$ value; thus, $\lambda_{\max }=69.11 \mathrm{kgf} / \mathrm{m}^{2}$. For the safe bearing capacity, the design wind force value $F(z=139 \mathrm{~m})_{\text {return }=10}$ was selected as the safe bearing capacity $\left(F_{\max }\right)$ value; thus, $F_{\max }=834 \mathrm{kN}$.

\subsection{Destruction and warning results}

In steps 10 and 11 of the procedures shown in Figure 12, the simulation results were evaluated regarding whether the scaffolds would be destructed and whether a warning would be needed within the next $6 \mathrm{~h}$. As shown in Table 3 the observed records were firstly analyzed to obtain the theoretical destruction information, including the destruction time point $t_{\text {collapse }}$ and the corresponding observed wind velocity $\left(V_{t_{\text {collapse }}}\right)$ and wind force $\left(F_{t_{\text {collapse }}}\right)$. Additionally, this study simulated the possible destruction analysis using the LSTM wind velocity prediction values, including the possible destruction time point $\left(t_{\text {collapse }}\right)$ and its corresponding predicted wind velocity $\left(V_{t_{\text {collapse }}}\right)$ and wind force $\left(F_{t_{\text {collapse }}}\right)$. The observed values of the three typhoon events indicated that the theoretical destruction caused by Typhoon Soudelor occurred at the 52th hour.

Results of the simulation conducted using the prediction values indicated that when the lead time was 1 or $2 \mathrm{~h}$, the destruction would occur at the 52th and 53th hours; no destruction was detected when the lead time is larger than $3 \mathrm{~h}$. Similar conditions were also detected in the simulations of Typhoons Dujuan and Megi; thus, for both typhoons, destruction could be detected when the lead time was equal to 1 and $2 \mathrm{~h}$, respectively. The longer the lead time in the prediction model was, the less accurate peak wind velocity predictions were. Therefore, the longer the forecasting horizon was, the more difficult it was to detect destruction.

An advanced warning mechanism was established to provide the construction contractors with more information about the wind velocity enhancement trends; the wind velocity alert value was defined as:

$$
V_{\text {alert }}=\mu \cdot V_{10}(C)_{\text {return }=10},
$$

where $\mu$ is the reduction factor. It was assumed that when $\mu=0.8, V_{\text {alert }}$ would be approximately $20 \mathrm{~m} / \mathrm{s}$.

As shown in Table 4, simulations were performed on the basis of data from the three typhoons: the warning time $\left(t_{\mathrm{cr}}\right)$, the corresponding wind velocity $\left(V_{t_{\mathrm{c}}}\right)$, and the corresponding wind force $\left(F_{t}\right)$. Additionally, this study also calculated how far in advance the system can issue a warning; this was calculated by subtracting the warning time $\left(t_{\mathrm{cr}}\right)$ from the theoretical destruction time point $\left(t_{\text {collapse }}\right)$.

The simulation results for the three typhoons indicated that for Typhoon Soudelor, the system could issue a warning when the lead time was equal to 1 to $6 \mathrm{~h}$, and an early warning could be issued 1 to $2 \mathrm{~h}$ in advance. For Typhoons Dujuan and Megi, the system could issue a warning, and the advanced warning of the system varied from 0 to $4 \mathrm{~h}$. 
a)

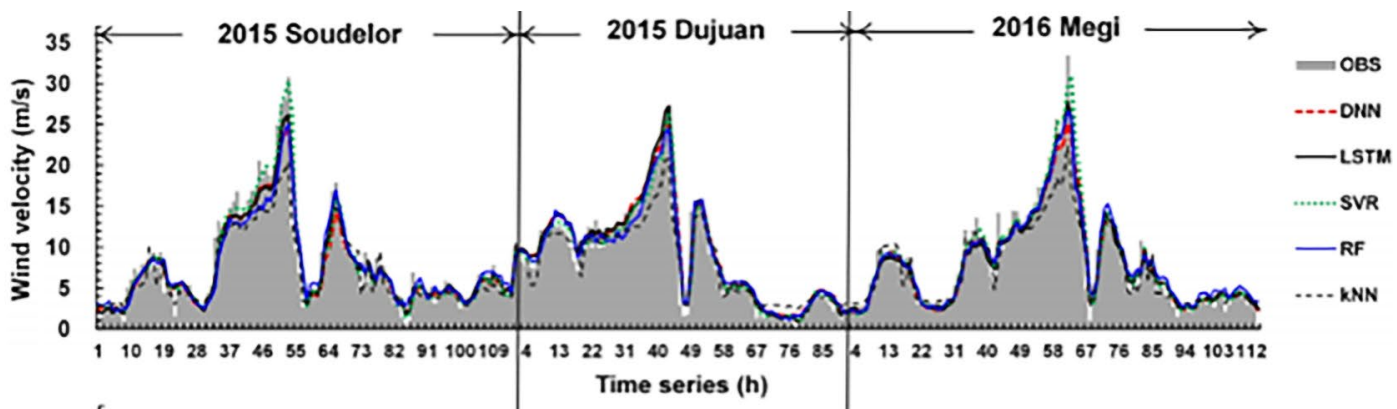

b)

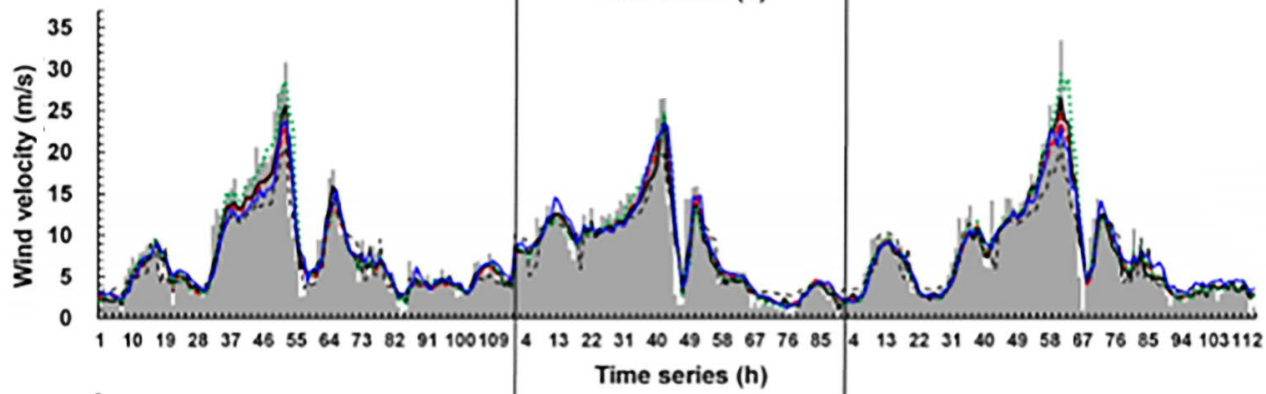

c)

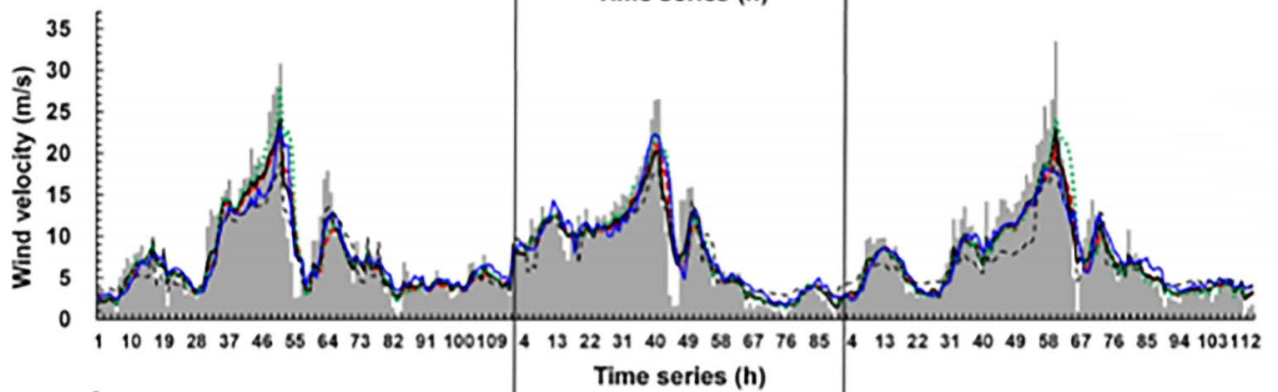

d)

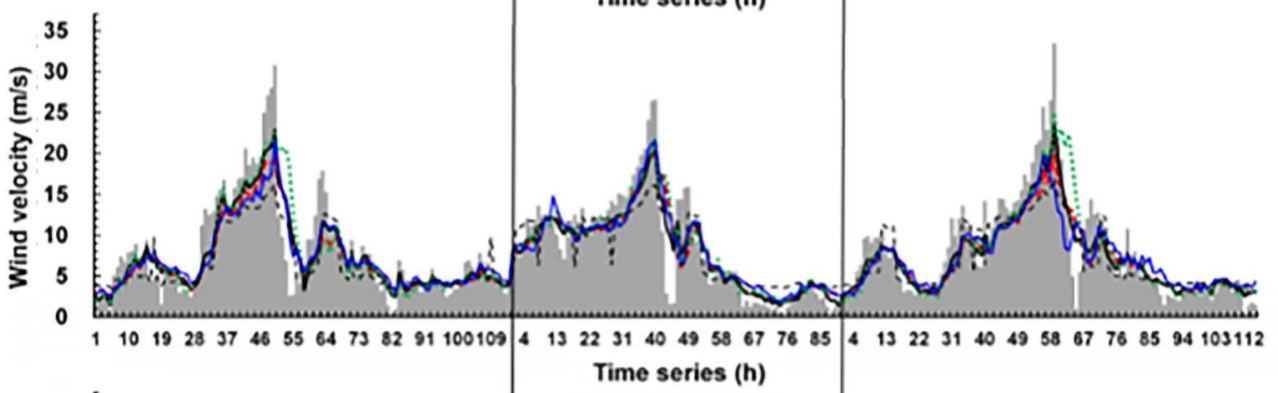

e)

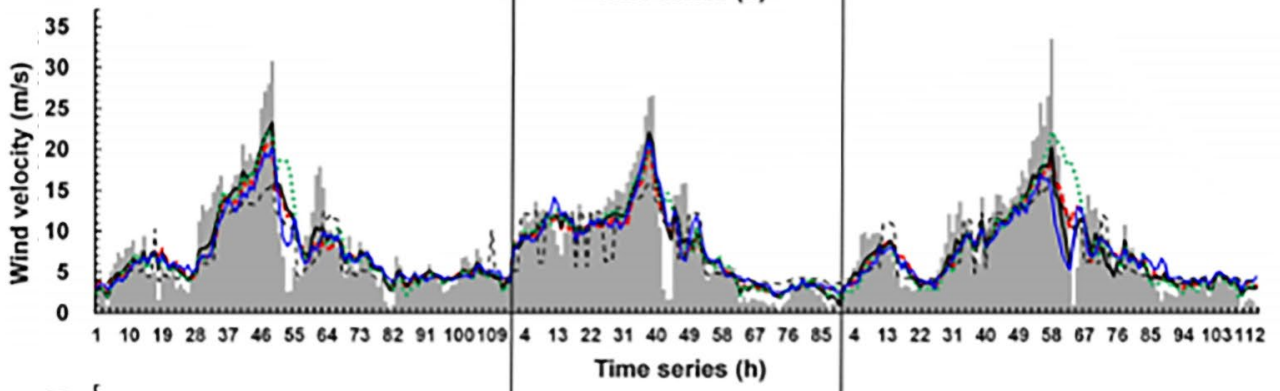

f)

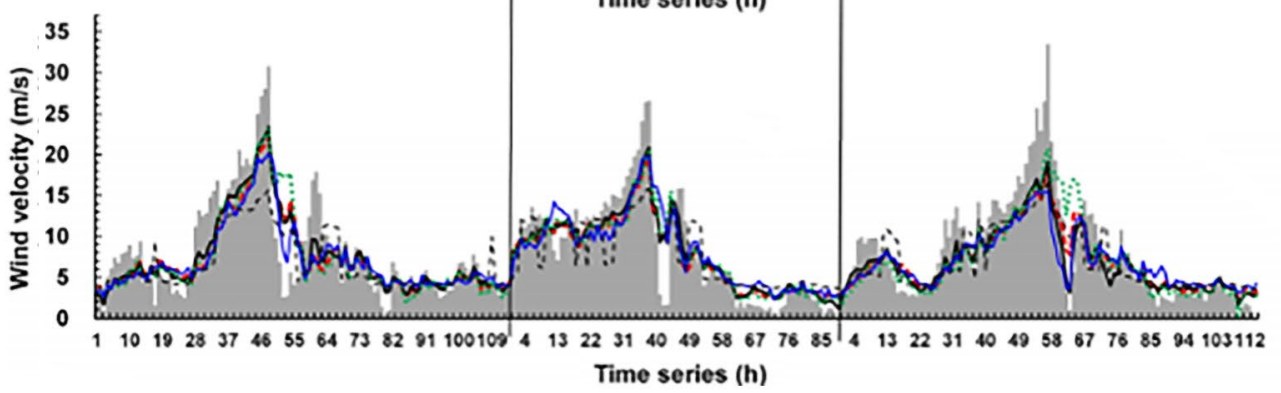

Figure 13. Predicted wind velocity graph for the three typhoons 


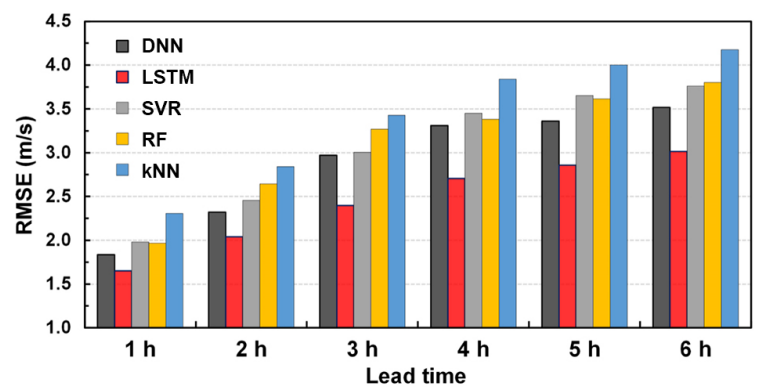

Figure 14. Performance levels of lead times 1-6 h using a testing set



Figure 15. The building $V-q-F$

Table 3. Simulation results for observation and prediction of potential collapse

\begin{tabular}{|c|c|c|c|c|c|c|c|}
\hline \multirow{2}{*}{ Typhoon } & \multirow{2}{*}{$\begin{array}{l}\text { Lead } \\
\text { time }\end{array}$} & \multicolumn{3}{|c|}{ Observation } & \multicolumn{3}{|c|}{ Prediction } \\
\hline & & $t_{\text {collapse }}(\mathrm{h})$ & $V_{t_{\text {collapse }}}(\mathrm{m} / \mathrm{s})$ & $F_{t_{\text {collapse }}}(\mathrm{kN})$ & $t_{\text {collapse }}(\mathrm{h})$ & $V_{t_{\text {collapse }}}(\mathrm{m} / \mathrm{s})$ & $F_{t_{\text {collapse }}}(\mathrm{kN})$ \\
\hline \multirow{6}{*}{ Soudelor } & $1 \mathrm{~h}$ & 52 & 27.1 & 948.5 & 53 & 25.7 & 851.5 \\
\hline & $2 \mathrm{~h}$ & 52 & 27.1 & 948.5 & 54 & 25.5 & 841.1 \\
\hline & $3 \mathrm{~h}$ & 52 & 27.1 & 948.5 & $\mathrm{~N} / \mathrm{A}$ & N/A & $\mathrm{N} / \mathrm{A}$ \\
\hline & $4 \mathrm{~h}$ & 52 & 27.1 & 948.5 & $\mathrm{~N} / \mathrm{A}$ & $\mathrm{N} / \mathrm{A}$ & N/A \\
\hline & $5 \mathrm{~h}$ & 52 & 27.1 & 948.5 & $\mathrm{~N} / \mathrm{A}$ & $\mathrm{N} / \mathrm{A}$ & $\mathrm{N} / \mathrm{A}$ \\
\hline & $6 \mathrm{~h}$ & 52 & 27.1 & 948.5 & N/A & N/A & $\mathrm{N} / \mathrm{A}$ \\
\hline \multirow{6}{*}{ Dujuan } & $1 \mathrm{~h}$ & 43 & 26.4 & 900.1 & 43 & 26.6 & 915.1 \\
\hline & $2 \mathrm{~h}$ & 43 & 26.4 & 900.1 & 43 & 25.4 & 833.2 \\
\hline & $3 \mathrm{~h}$ & 43 & 26.4 & 900.1 & $\mathrm{~N} / \mathrm{A}$ & N/A & N/A \\
\hline & $4 \mathrm{~h}$ & 43 & 26.4 & 900.1 & $\mathrm{~N} / \mathrm{A}$ & $\mathrm{N} / \mathrm{A}$ & $\mathrm{N} / \mathrm{A}$ \\
\hline & $5 \mathrm{~h}$ & 43 & 26.4 & 900.1 & $\mathrm{~N} / \mathrm{A}$ & N/A & N/A \\
\hline & $6 \mathrm{~h}$ & 43 & 26.4 & 900.1 & $\mathrm{~N} / \mathrm{A}$ & $\mathrm{N} / \mathrm{A}$ & $\mathrm{N} / \mathrm{A}$ \\
\hline \multirow{6}{*}{ Megi } & $1 \mathrm{~h}$ & 62 & 26.5 & 906.9 & 63 & 27.7 & 992.1 \\
\hline & $2 \mathrm{~h}$ & 62 & 26.5 & 906.9 & 63 & 26.5 & 908.9 \\
\hline & $3 \mathrm{~h}$ & 62 & 26.5 & 906.9 & $\mathrm{~N} / \mathrm{A}$ & $\mathrm{N} / \mathrm{A}$ & N/A \\
\hline & $4 \mathrm{~h}$ & 62 & 26.5 & 906.9 & N/A & N/A & N/A \\
\hline & $5 \mathrm{~h}$ & 62 & 26.5 & 906.9 & N/A & N/A & N/A \\
\hline & $6 \mathrm{~h}$ & 62 & 26.5 & 906.9 & N/A & N/A & N/A \\
\hline
\end{tabular}

Table 4. Simulation results for a warning when the wind alert value is at $20 \mathrm{~m} / \mathrm{s}$

\begin{tabular}{|c|c|c|c|c|c|c|c|}
\hline \multirow{2}{*}{ Typhoon } & \multirow{2}{*}{$\begin{array}{l}\text { Lead } \\
\text { time }\end{array}$} & \multicolumn{2}{|c|}{ Observation } & \multicolumn{3}{|c|}{ Prediction } & \multirow{2}{*}{$\begin{array}{l}\text { Length of time for } \\
\text { early warning }(\mathrm{h})\end{array}$} \\
\hline & & $t_{\text {collapse }}(\mathrm{h})$ & $V_{t_{\text {collapse }}}(\mathrm{m} / \mathrm{s})$ & $t_{\mathrm{cr}}(\mathrm{h})$ & $V_{t_{\mathrm{cr}}}(\mathrm{m} / \mathrm{s})$ & $F_{t_{\mathrm{cr}}}(\mathrm{kN})$ & \\
\hline \multirow{6}{*}{ Soudelor } & $1 \mathrm{~h}$ & 52 & 27.1 & 50 & 20.4 & 536.6 & 2 \\
\hline & $2 \mathrm{~h}$ & 52 & 27.1 & 50 & 20.9 & 564.5 & 2 \\
\hline & $3 \mathrm{~h}$ & 52 & 27.1 & 51 & 20.5 & 544.2 & 1 \\
\hline & $4 \mathrm{~h}$ & 52 & 27.1 & 51 & 20.1 & 519.5 & 1 \\
\hline & $5 \mathrm{~h}$ & 52 & 27.1 & 51 & 20.4 & 535.8 & 1 \\
\hline & $6 \mathrm{~h}$ & 52 & 27.1 & 51 & 20.6 & 550.1 & 1 \\
\hline \multirow{6}{*}{ Dujuan } & $1 \mathrm{~h}$ & 43 & 26.4 & 39 & 20.0 & 516.6 & 4 \\
\hline & $2 \mathrm{~h}$ & 43 & 26.4 & 41 & 22.1 & 630.0 & 2 \\
\hline & $3 \mathrm{~h}$ & 43 & 26.4 & 41 & 20.2 & 524.5 & 2 \\
\hline & $4 \mathrm{~h}$ & 43 & 26.4 & 42 & 20.0 & 514.3 & 1 \\
\hline & $5 \mathrm{~h}$ & 43 & 26.4 & 42 & 20.1 & 521.8 & 1 \\
\hline & $6 \mathrm{~h}$ & 43 & 26.4 & 43 & 20.0 & 517.0 & 0 \\
\hline \multirow{6}{*}{ Megi } & $1 \mathrm{~h}$ & 62 & 26.5 & 59 & 21.0 & 567.2 & 3 \\
\hline & $2 \mathrm{~h}$ & 62 & 26.5 & 59 & 20.1 & 519.7 & 3 \\
\hline & $3 \mathrm{~h}$ & 62 & 26.5 & 60 & 20.3 & 530.4 & 2 \\
\hline & $4 \mathrm{~h}$ & 62 & 26.5 & 60 & 20.0 & 516.6 & 2 \\
\hline & $5 \mathrm{~h}$ & 62 & 26.5 & 61 & 20.2 & 527.3 & 1 \\
\hline & $6 \mathrm{~h}$ & 62 & 26.5 & 62 & 20.0 & 516.6 & 0 \\
\hline
\end{tabular}


Of course, when the warning time length was equal to 0 $\mathrm{h}$, the warning was issued simultaneously to destruction. The $\mu$ value could be adjusted accordingly to make the warning time longer.

\section{Discussion}

This study demonstrated a methodology for developing a usable TISCEWS system for evaluating structural stress on scaffolds from the wind force and determining the destruction time point in the future. The TISCEWS system developed in the study contains three modules. The first module involves the establishment of wind velocity prediction models, the second module contains the analysis of the wind force on the scaffolds, and the third module involves the development of the scaffold collapse evaluation approach.

For the first module, to provide an accurate wind velocity prediction for operating the TISCEWS warning system, this study established the wind velocity prediction models using DL and ML techniques. The application of the LSTM model enhanced the forecasting ability of the wind velocity. Because the LSTM is a neural network containing memory cell blocks, each memory cell block comprises memory cell units that retain states across timesteps as well as three gate units (input gate, output gate, and forget gate). A set of gates is used to control the input, output, and storage of information. This architecture allows learning of long-term dependencies (Monner \& Reggia, 2012; Schmidhuber, 2015). Therefore, the length of time window is determined based on the special units of memory blocks (i.e., adaptively forgetting or resetting cell memory). LSTM recurrent neural networks are capable of learning and remembering long time sequences of inputs (Sak et al., 2014; Wei, 2020). The wind prediction results revealed that the LSTM model could yield higher accuracy than the DNN, SVR, RF, and $k \mathrm{NN}$ models. To compare the prediction accuracy of DL-based and ML-based models, first we compute the average RMSE measures for 1-6 h predictions using three testing typhoons (i.e., Soudelor, Dujuan, and Megi). After calculation, the average RMSE values of DNN, LSTM, SVR, RF, and $k N N$ were 2.886, $2.444,3.051,3.113$, and $3.432 \mathrm{~m} / \mathrm{s}$, respectively. Then, we define the improvement rate to compare these models. The improvement rate of RMSE, $\mathrm{IR}_{\mathrm{RMSE}}$, is given as

$\operatorname{IR}_{\mathrm{RMSE}}(\%)=\left(\mathrm{RMSE}_{\max }-\mathrm{RMSE}_{i}\right) / \mathrm{RMSE}_{\text {max }} \times 100,(17)$

where $\mathrm{RMSE}_{\max }$ is the maximal average RMSE value of all models, and $\mathrm{RMSE}_{i}$ is the average RMSE value at model $i$. The higher the $\operatorname{IR}_{\mathrm{RMSE}}$ value is, the more accurate the performance of the predicted outcomes is.

Here, the RMSE $E_{\max }$ can be the average RMSE of $k \mathrm{NN}$ model. Then, the DNN, LSTM, SVR, and RF models revealed respective improvements of $15.90 \%, 28.79 \%$, $11.11 \%$, and $9.28 \%$ compared with the $k \mathrm{NN}$ model. By examining the $\mathrm{IR}_{\mathrm{RMSE}}$ results of all models, we determined that the improvement metric resulting from the use of
LSTM was higher than that from the use of DNN, SVR, $\mathrm{RF}$ and $k \mathrm{NN}$.

It is noted that this study used the trial-and-error method to calibrate the model hyperparameters. Future studies should conduct an optimization algorithm, such as the evolutionary optimization (Liang et al. 2001; Tan et al., 2003), to increase the ability to determine global optimal parameter values, although this is generally at the expense of computational efficiency.

Le Méridien Taichung, Taiwan, was used as the experimental site for the second module. In practice, the proposed methodology can be used for determining whether the scaffold structure can withstand the stress of the wind force. However, various construction projects may not have the same scaffold construction conditions as the experimental site. Therefore, when using the proposed methodology in such construction projects, some parameters used in the SCAFFOLDS model should be tuned to suit the local construction conditions. For instance, the characteristic area of the scaffolds, wind force coefficient of wind effects, and centroid elevation of characteristic area should be reset.



Figure 16. Different reduction rate

For the third module, to investigate the effect of the reduction factor on the length of time for early warning, $\mu$ values ranging from 0.6 to 0.8 were tested. Figure 16 illustrates the average lengths of time for early warning by using three testing typhoons. As the reduction factor decreases, the length of time for early warning increases, because the lower the wind velocity alert values are, the easier the wind velocity predictions are. However, the requirements of the construction units should be considered when determining a suitable $\mu$ value, and the set $\mu$ value cannot be overly small. An overly small $\mu$ value could result in frequent warnings, which would defeat the purpose of the warning system.

\section{Conclusions}

The frame-type scaffolds used during construction of buildings in Taiwan have occasionally been found prone to collapse during typhoons. For the construction industry, timely and reliable information on recent, current, and future wind speeds are thus vital to enable forecasters to make accurate and timely forecasts and for construction sites to be managed appropriately. Because construction 
often fails during the construction stage under the influence of wind, a useful scheme for wind speed forecasting during typhoon periods is highly desired. Hence, the study on wind speeds during typhoons is of great significance for the construction industry and operations management.

This study proposed an early warning system for scaffold collapse caused by typhoon winds. The TISCEWS was proposed to evaluate the structural stress on scaffolds from wind force and determine the destruction time point in the future for the construction unit. Three modules were involved in establishing the early warning system. The study area was Taichung City, Taiwan. The wind velocity prediction was performed using the DL-based and MLbased models. The results indicated that the LSTM model outperformed the DNN, SVR, RF and $k \mathrm{NN}$ models across all lead times (i.e., $1-6 \mathrm{~h}$ ). Therefore, this study adopted the LSTM prediction values when simulating the destruction analysis for scaffolds. Three typhoons (Soudelor in 2015, Dujuan in 2015, and Megi in 2016) were selected as the test cases. In terms of the wind force destruction analysis for scaffolds, the results revealed that the system successfully output the possible collapse time for scaffolds within 1 to $6 \mathrm{~h}$; the setting of wind velocity alert values also effectively advanced the warning time. Finally, the achievements of this study can enhance the ability of engineers to assess the wind resistance of scaffolds. By finishing the warning system for scaffold collapse caused by typhoon winds, engineers can reinforce the structure, preventing the collapse of scaffolding and reducing wind accidents.

\section{Acknowledgements}

The author is grateful for the data provided by the Central Weather Bureau of Taiwan.

\section{Funding}

The work was supported by the Ministry of Science and Technology, Taiwan [number MOST108-2622-M-019001-CC3].

\section{Author contributions}

Chih-Chiang WEI conceived the study and was responsible for the design and development of the methodology and data analysis, and writing-original draft.

\section{Disclosure statement}

Article does not have any competing financial, professional, or personal interests from other parties.

\section{References}

Baudron, P., Alonso-Sarría, F., García-Aróstegui, J. L., Cánovas-García, F., Martínez-Vicente, D., \& Moreno-Brotóns, J. (2013). Identifying the origin of groundwater samples in a multi-layer aquifer system with Random Forest classification. Journal of Hydrology, 499, 303-315.

https://doi.org/10.1016/j.jhydrol.2013.07.009

Beli, I. L. K., \& Guo, C. (2017). Enhancing face identification using local binary patterns and k-nearest neighbors. Journal of Imaging, 3, 37. https://doi.org/10.3390/jimaging3030037

Brandt, M., Grau, T., Mbow, C., \& Samimi, C. (2014). Modeling soil and woody vegetation in the Senegalese Sahel in the context of environmental change. Land, 3, 770-792. https://doi.org/10.3390/land3030770

Breiman, L. (2001). Random Forests. Machine Learning, 45, 5-32. https://doi.org/10.1023/A:1010933404324

Byeon, W., Liwicki, M., \& Breuel, T. M. (2015). Scene analysis by mid-level attribute learning using 2D LSTM networks and an application to web-image tagging. Pattern Recognition Letters, 63, 23-29. https://doi.org/10.1016/j.patrec.2015.06.003

Cadenas, E., \& Rivera, W. (2010). Wind speed forecasting in three different regions of Mexico, using a hybrid ARIMAANN model. Renewable Energy, 35, 2732-2738. https://doi.org/10.1016/j.renene.2010.04.022

Cadenas, E., Rivera, W., Campos-Amezcua, R., \& Heard, C. (2016). Wind speed prediction using a univariate ARIMA model and a multivariate NARX model. Energies, 9, 109. https://doi.org/10.3390/en9020109

Chen, J., Zeng, G., Zhou, W., Du, W., \& Lu, K. (2018). Wind speed forecasting using nonlinear-learning ensemble of deep learning time series prediction and extremal optimization. Energy Conversion and Management, 165, 681-695. https://doi.org/10.1016/j.enconman.2018.03.098

Chen, K. Y., \& Wang, C. H. (2007). Support vector regression with genetic algorithms in forecasting tourism demand. Tourism Management, 28, 215-226. https://doi.org/10.1016/j.tourman.2005.12.018

Cheng, C. C., Hsu, N. S., \& Wei, C. C. (2008). Decision-tree analysis on optimal release of reservoir storage under typhoon warnings. Natural Hazards, 44, 65-84. https://doi.org/10.1007/s11069-007-9142-1

Chou, J. S., Truong, D. N., \& Che, Y. (2020). Optimized multioutput machine learning system for engineering informatics in assessing natural hazards. Natural Hazards, 101, 727-754. https://doi.org/10.1007/s11069-020-03892-2

Chung, J., Gulcehre, C., Cho, K., \& Bengio, Y. (2014). Empirical evaluation of gated recurrent Neural networks on sequence modeling. In NIPS 2014 Deep Learning and Representation Learning Workshop. https://arxiv.org/abs/1412.3555v1

Cristianini, N., \& Shawe-Taylor, J. (2000). An introduction to support vector machines and other Kernel-based learning methods. Cambridge: Cambridge University Press. https://doi.org/10.1017/CBO9780511801389

Cutler, D., Edwards, T. C., Beard, K. H., Cutler, A., Hess, K. T., Gibson, J., \& Lawler, J. J. (2007). Random forest for classification in ecology. Ecology, 88, 2783-2792.

https://doi.org/10.1890/07-0539.1

Dongmei, H., Shiqing, H., Xuhui, H., \& Xue, Z. (2017). Prediction of wind loads on high-rise building using a BP neural network combined with POD. Journal of Wind Engineering \& Industrial Aerodynamics, 170, 1-17. https://doi.org/10.1016/j.jweia.2017.07.021

Du, J., \& Xu, Y. (2017). Hierarchical deep neural network for multivariate regression. Pattern Recognition, 63, 149-157. https://doi.org/10.1016/j.patcog.2016.10.003

Fix, E., \& Hodges, J. L. (1951). Discriminatory analysis, nonaparametric discrimination: Consistency properties (Technical Report 4). USAF School of Aviation Medicine, Randolph Field. https://doi.org/10.1037/e471672008-001 
Glüge, S., Böck, R., Palm, G., \& Wendemuth, A. (2014). Learning long-term dependencies in segmented-memory recurrent neural networks with backpropagation of error. Neurocomputing, 141, 54-64. https://doi.org/10.1016/j.neucom.2013.11.043

Graves, A. (2012). Supervised sequence labelling with recurrent neural networks (vol. 385). Springer.

https://doi.org/10.1007/978-3-642-24797-2

Graves, A. (2013). Generating sequences with recurrent neural networks. https://arxiv.org/abs/1308.0850v5

Graves, A., \& Schmidhuber, J. (2005). Framewise phoneme classification with bidirectional LSTM and other neural network architectures. Neural Networks, 18, 602-610. https://doi.org/10.1016/j.neunet.2005.06.042

Hochreiter, S., \& Schmidhuber, J. (1997). Long short-term memory. Neural Computation, 9, 1735-1780.

https://doi.org/10.1162/neco.1997.9.8.1735

Hu, Q., Zhang, R., \& Zhou, Y. (2016). Transfer learning for shortterm wind speed prediction with deep neural networks. Renewable Energy, 85, 83-95.

https://doi.org/10.1016/j.renene.2015.06.034

Huang, C. J., \& Kuo, P. H. (2018). A short-term wind speed forecasting model by using artificial neural networks with stochastic optimization for renewable energy systems. Energies, 11, 2777. https://doi.org/10.3390/en11102777

Huang, X., Gao, L., Crosbie, R. S., Zhang, N., Fu, G., \& Doble, R. (2019). Groundwater recharge prediction using linear regression, multi-layer perception network, and deep learning. $W a-$ ter, 11, 1879. https://doi.org/10.3390/w11091879

Huang, Y., Jin, L., Zhao, H., \& Huang, X. (2018a). Fuzzy neural network and LLE Algorithm for forecasting precipitation in tropical cyclones: comparisons with interpolation method by ECMWF and stepwise regression method. Natural Hazards, 91, 201-220. https://doi.org/10.1007/s11069-017-3122-x

Huang, Y., Liu, S., \& Yang, L. (2018b). Wind speed forecasting method using EEMD and the combination forecasting method based on GPR and LSTM. Sustainability, 10, 3693. https://doi.org/10.3390/su10103693

Kim, M., Park, M., Im, J., Park, S., \& Lee, M. I. (2019). Machine learning approaches for detecting tropical cyclone formation using satellite data. Remote Sensing, 11, 1195.

https://doi.org/10.3390/rs11101195

Kingma, D. P., \& Ba, J. L. (2015). ADAM: A method for stochastic optimization. In International Conference on Learning Representations (ICLR 2015).

Liang, K. H., Yao, X., \& Newton, C. S. (2001). Adapting self-adaptive parameters in evolutionary algorithms. Applied Intelligence, 15, 171-180. https://doi.org/10.1023/A:1011286929823

Lin, C. C., \& Yen, C. (2016). Research on the safety performance influence factors and safety design key points of scaffolding (Report No. ILOSH104-S310). Institute of Labor, Occupational Safety and Health, Ministry of Labor, Taiwan (in Chinese).

Lin, C. C., \& Yen, C. (2017). Study on wind accidents and wind loads of facade frame type scaffolds (Report No. ILOSH105S307). Institute of Labor, Occupational Safety and Health, Ministry of Labor, Taiwan (in Chinese).

Lipton, Z. C., Berkowitz, J., \& Elkan, C. (2015). A critical review of recurrent neural networks for sequence learning. https://arxiv.org/abs/1506.00019v4

Liu, H., Mi, X. W., \& Li, Y. F. (2018). Wind speed forecasting method based on deep learning strategy using empirical wavelet transform, long short term memory neural network and Elman neural network. Energy Conversion and Management, 156, 498-514.

https://doi.org/10.1016/j.enconman.2017.11.053
Lu, W., Zhang, Y., Xu, C., Lin, C., \& Huo, Y. (2019). A deep learning-based satellite target recognition method using radar data. Sensors, 19, 2008. https://doi.org/10.3390/s19092008

Mallick, M., Mohanta, A., Kumar, A., \& Patra, K. C. (2020). Prediction of wind-induced mean pressure coefficients using GMDH neural network. Journal of Aerospace Engineering, 33, 04019104.

https://doi.org/10.1061/(ASCE)AS.1943-5525.0001101

Masetic, Z., \& Subasi, A. (2016). Congestive heart failure detection using random forest classifier. Computer Methods and Programs in Biomedicine, 130, 54-64.

https://doi.org/10.1016/j.cmpb.2016.03.020

Ministry of the Interior. (2014). Building technical regulations (Act No. 1020812044). Taiwan (in Chinese).

Ministry of the Interior. (2015). Wind resistance design specifications and commentary of buildings (Act No. 1030805400). Taiwan (in Chinese).

Ministry of Labor. (2014). Establish safety and health facilities standards (Act No. 10302006411). Taiwan (in Chinese).

Monner, D., \& Reggia, J. A. (2012). A generalized LSTM-like training algorithm for second-order recurrent. Neural Networks, 25, 70-83. https://doi.org/10.1016/j.neunet.2011.07.003

Nair, V., \& Hinton, G. (2010). Rectified linear units improve restricted Boltzmann machines. In Proceedings of the 27th International Conference on Machine Learning (pp. 807-814), Haifa, Israel.

Noorollahi, Y., Jokar, M., \& Kalhor, A. (2016). Using artificial neural networks for temporal and spatial wind speed forecasting in Iran. Energy Conversion and Management, 115, 17-25. https://doi.org/10.1016/j.enconman.2016.02.041

Pal, M. (2005). Random forest classifier for remote sensing classification. International Journal of Remote Sensing, 26, 217222. https://doi.org/10.1080/01431160412331269698

Panapakidis, I. P., Michailides, C., \& Angelides, D. C. (2019). A data-driven short-term forecasting model for offshore wind speed prediction based on computational intelligence. Electronics, 8, 420. https://doi.org/10.3390/electronics 8040420

Sak, H., Senior, A., \& Beaufays, F. (2014). Long short-term memory recurrent neural network architectures for large scale acoustic modeling. In Proceedings of the Annual Conference of International Speech Communication Association (INTERSPEECH).

Schmidhuber, J. (2015). Deep learning in neural networks: An overview. Neural Networks, 61, 85-117.

https://doi.org/10.1016/j.neunet.2014.09.003

Sheela, K. G., \& Deepa, S. N. (2013). Neural network based hybrid computing model for wind speed prediction. Neurocomputing, 122, 425-429.

https://doi.org/10.1016/j.neucom.2013.06.008

Shi, X., Lei, X., Huang, Q., Huang, S., Ren, K., \& Hu, Y. (2018). Hourly day-ahead wind power prediction using the hybrid model of variational model decomposition and long shortterm memory. Energies, 11, 3227.

https://doi.org/10.3390/en11113227

Tan, K. C., Khor, E. F., Lee, T. H., \& Sathikannan, R. (2003). An evolutionary algorithm with advanced goal and priority specification for multi-objective optimization. Journal of Artificial Intelligence Research, 18, 183-215.

https://doi.org/10.1613/jair.842

Üstün, B., Melssen, W. J., Oudenhuijzen, M., \& Buydens, L. M. C. (2005). Determination of optimal support vector regression parameters by genetic algorithms and simplex optimization. Analytica Chimica Acta, 544, 292-305.

https://doi.org/10.1016/j.aca.2004.12.024 
Vapnik, V. (1995). The nature of statistical learning theory. Springer-Verlag.

https://doi.org/10.1007/978-1-4757-2440-0

Wei, C. C. (2012). Wavelet kernel support vector machines forecasting techniques: case study on water-level predictions during typhoons. Expert Systems with Applications, 39, 51895199. https://doi.org/10.1016/j.eswa.2011.11.020

Wei, C. C. (2014). Surface wind nowcasting in the Penghu Islands based on classified typhoon tracks and the effects of the Central Mountain Range of Taiwan. Weather and Forecasting, 29, 1425-1450. https://doi.org/10.1175/WAF-D-14-00027.1

Wei, C. C. (2015). Forecasting surface wind speeds over offshore islands near Taiwan during tropical cyclones: comparisons of data-driven algorithms and parametric wind representations. Journal of Geophysical Research: Atmospheres, 120, 1826-1847. https://doi.org/10.1002/2014JD022568

Wei, C. C. (2017). Conceptual weather environmental forecasting system for identifying potential failure of under-construction structures during typhoons. Journal of Wind Engineering and Industrial Aerodynamics, 168, 48-59.

https://doi.org/10.1016/j.jweia.2017.05.010

Wei, C. C. (2019). Study on wind simulations using deep learning techniques during typhoons: a case study of Northern Taiwan. Atmosphere, 10, 684. https://doi.org/10.3390/atmos 10110684

Wei, C. C. (2020). Comparison of river basin water level forecasting methods: sequential neural networks and multiple-input functional neural networks. Remote Sensing, 12, 4172. https://doi.org/10.3390/rs12244172

Weninger, F., Geiger, J., Wöllmer, M., Schuller, B., \& Rigoll, G. (2014). Feature enhancement by deep LSTM networks for ASR in reverberant multisource environments. Computer Speech and Language, 28, 888-902. https://doi.org/10.1016/j.csl.2014.01.001

Wollmer, M., Eyben, F., Graves, A., Schuller, B., \& Rigoll, G. (2010). Bidirectional LSTM networks for context-sensitive keyword detection in a cognitive virtual agent framework. Cognitive Computation, 2, 180-190. https://doi.org/10.1007/s12559-010-9041-8

Wollmer, M., Schuller, B., \& Rigoll, G. (2013). Keyword spotting exploiting long short-term memory. Speech Communication, 55, 252-265. https://doi.org/10.1016/j.specom.2012.08.006

Yao, C., Cai, D., Bu, J., \& Chen, G. (2017). Pre-training the deep generative models with adaptive hyperparameter optimization. Neurocomputing, 247, 144-155. https://doi.org/10.1016/j.neucom.2017.03.058

Zhang, Y., Wang, X., \& Tang, H. (2019). An improved Elman neural network with piecewise weighted gradient for time series prediction. Neurocomputing, 359, 99-208.

https://doi.org/10.1016/j.neucom.2019.06.001 